\title{
Inhibitory effects of forkhead box L1 gene on osteosarcoma growth through the induction of cell cycle arrest and apoptosis
}

\author{
XIAODONG CHEN ${ }^{1}$, MIN DENG $^{2}$, LI MA ${ }^{3}$, JIANSHENG ZHOU ${ }^{1}$, YUZHOU XIAO ${ }^{1}$, \\ XINSHE ZHOU ${ }^{1}, \mathrm{CHANGCHUN} \mathrm{ZHANG}^{1}$ and MIN WU ${ }^{1}$ \\ Departments of ${ }^{1}$ Orthopedics, ${ }^{2}$ Gastroenterology and ${ }^{3}$ Pathology, The First Affiliated Hospital \\ of Bengbu Medical College, Bengbu, Anhui, P.R. China
}

Received December 29, 2014; Accepted March 19, 2015

DOI: $10.3892 /$ or.2015.3969

\begin{abstract}
Forkhead box L1 (FOXL1), which is considered a novel candidate tumor suppressor, inhibits proliferation and invasion in certain types of cancer. However, the regulation and function of FOXL1 in osteosarcoma remains unclear. The expression of FOXL1 gene in osteosarcoma tissues and cell lines was examined using RT-PCR, immunohistochemistry and western blot analysis. pcDNA-FOXL1 carrying full-length FOXL1 cDNA was constructed to upregulate the level of FOXL1 expression in osteosarcoma cell lines. The proliferation, cell cycle and apoptosis of osteosarcoma cells in vitro or in vivo were examined following transfection with pcDNA-FOXL1. In addition, the expression of p21, p27, cytochrome $c$ and caspase-3, which may be involved in the regulation of the cell cycle and apoptosis was examined using western blot analysis. The results showed that FOXL1 expression was downregulated in osteosarcoma tissues and cell lines. Loss or downregulation of FOXL1 was associated with poor prognosis. Ectopic FOXL1 expression inhibited cell proliferation in vitro and in vivo. The ectopic FOXL1 expression increased the expression of p21 and p27, which induced G1 arrest in the U-2 OS cells. In addition, the ectopic FOXL1 expression induced cytochrome $c$ release between mitochondria and cytoplasm, which disrupted the mitochondrial transmembrane potential and triggered intrinsic pathway apoptosis. In conclusion, the downregulation of FOXL1 expression was associated with osteosarcoma cell growth. Restoration of FOXL1 gene expression by gene therapy may have a therapeutic potential for patients with osteosarcoma.
\end{abstract}

Correspondence to: Dr Min Deng, Department of Gastroenterology, The First Affiliated Hospital of Bengbu Medical College, 287 Changhuai Road, Bengbu, Anhui, P.R. China

E-mail:dm8558@yeah.net

Key words: FOXL1, osteosarcoma, apoptosis, cell cycle

\section{Introduction}

Osteosarcoma, the most common primary bone malignancy, is most prevalent in children and young adults (1). Due to its high propensity for local invasion, early metastasis and recurrence, osteosarcoma leads to a high mortality rate. It has been documented that the 3-year disease-free survival of osteosarcoma patients remains 60-70\% despite combined therapy including surgery, chemotherapy and radiotherapy (2). The molecular mechanism underlying osteosarcoma development and metastasis has yet to be fully elucidated, which has a negative impact on the improvement of diagnosis and treatment. Therefore, an improved understanding of the molecular biology and carcinogenic mechanism underlying the development and progression of osteosarcoma may be useful in the treatment of this malignancy.

The forkhead box (FOX) superfamily comprises a number of transcription factors which share a highly conserved DNA-binding domain (forkhead/winged helix-box) that plays a role in the regulation of various processes, including metabolism, cell proliferation and gene expression during ontogenesis (3). The role of FOX proteins in various types of cancer has been comprehensively investigated considering that these proteins have vital functions on biological processes. Certain Fox members including FOXP1, FOXM1, FOXR1 and FOXA1, show a diversity of activities on tumorigenesis and progression of cancer (4-8), while having dual roles in cancer biology. For instance, on the one hand, FOXP1 overexpression by chromosome translocations is associated with poor prognosis in lymphomas, suggesting it acts as an oncogene (4). On the other hand, loss of FOXP1 expression is associated with poor prognosis in breast cancer, suggesting FOXP1 also acts as a tumor suppressor in other types of cancer (5). As tumor suppressors or oncogenes, Fox proteins may function as direct or indirect targets of therapeutic intervention.

The present study focused on FOXL1 which belongs to the FOXL subfamily. As a novel identified tumor suppressor, the re-expression or overexpression of FOXL1 inhibits growth and induces apoptosis in colonic, pancreatic and gallbladder cancer cells (9-11). However, the role of FOXL1 in osteosarcoma remains to be investigated. In the present study, the association of FOXL1 expression with prognosis of osteosarcoma patients was assessed. In addition, the biological functions of FOXL1 
in proliferation, cell cycle and apoptosis of osteosarcoma cells were evaluated in vitro or in vivo.

\section{Materials and methods}

Patients and tissue collections. The study was approved by the Ethics Committee of The First Affiliated Hospital of Bengbu Medical College and written informed consent was obtained from all the patients. Thirty-seven of the patients with osteosarcoma enrolled in the present study were treated with radical resection (without prior radiotherapy or chemotherapy) between 2007 and 2009 at the Department of Orthopedics, The First Affiliated Hospital of Bengbu Medical College. Specimens of tumor tissues $(n=37)$ and adjacent non-tumor tissues $(n=18)$ were collected immediately following surgery, fixed with formalin and then embedded with paraffin. The specimens were diagnosed by two pathologists. Following treatment with the same chemotherapy regimens, a follow-up was performed for all the patients.

Immunohistochemistry. Serial sections $(5-\mu \mathrm{m})$ were cut from formalin-fixed, paraffin-embedded tumor or non-tumor blocks. The sections were dewaxed in xylene and rehydrated with concentration gradients. Heat-induced antigen retrieval was performed in a conventional pressure cooker, followed by elimination of endogenous peroxidase activity with $\mathrm{H}_{2} \mathrm{O}_{2}$. To decrease non-specific binding and background staining, the sections were incubated with $10 \%$ goat serum in phosphate-buffered saline (PBS). The sections were then incubated with primary antibodies against FOXL1 (1:100 dilution) overnight at $4{ }^{\circ} \mathrm{C}$ and then incubated with a secondary antibody (1:500 dilution) (both from Santa Cruz Biotechnology, Inc., Santa Cruz, CA, USA) at room temperature for $2 \mathrm{~h}$. The horseradish peroxidase-conjugated antibody was detected and visualized by 3,3'-diaminobenzidine tetrachloride (DAB). Immunostained sections were blindly reviewed by two independent pathologists. The staining intensity was scored using a 3 -scale system $(0,-$ or negative; $1,+$ or weak; $2,++$ or moderate; and $3,+++$ or strong) and osteosarcoma patients were classified into two groups according to the scores of FOXL1 immunostaining: low expression (-or + ) and high expression $(++$ or +++$)$.

Cell lines and culture conditions. Osteosarcoma cell lines U-2 OS, MG-63 and Saos-2 were all purchased from the Cell Bank of the Chinese Academy of Sciences, Shanghai, China. All cell lines were cultured in Dulbecco's modified Eagle's medium (DMEM; Invitrogen, Carlsbad, CA, USA) supplemented with $10 \%$ (v/v) fetal bovine serum (FBS; HyClone, Logan, UT, USA) and 1\% penicillin/streptomycin (Invitrogen) at $37^{\circ} \mathrm{C}$ in a humidified $5 \% \mathrm{CO}_{2}$ incubator.

Transfections. The pcDNA-FOXL1 carrying full-length of FOXL1 cDNA and the pcDNA3.1 empty vector were generous gifts from Dr Qin (Department of General Surgery, Xinhua Hospital, Affiliated to School of Medicine, Shanghai Jiaotong University, Shanghai, China). pcDNA-FOXL1 was transfected into osteosarcoma cells to restore or upregulate the FOXL1 expression. Transfected cells were cultured with medium supplemented with geneticin (G418, 200-300 $\mu \mathrm{g} / \mathrm{ml})$ for
10-14 days to generate stable transfectants. The expression of FOXL1 in transfected cells was examined by RT-PCR and western blot analysis.

RT-PCR assay. Reverse transcription-polymerase chain reaction (RT-PCR) was used to examine the FOXL1 mRNA expression in Saos-2, MG-63 and U-2 OS cells. Total RNA was extracted from the cell lines using a TRIzol reagent kit (Gibco-BRL, Gaithersburg, MD, USA) according to the manufacturer's instructions. After quantification, a certain amount of RNAs was reverse transcribed into cDNA using a PrimeScript ${ }^{\mathrm{TM}}$ RT-PCR kit (Takara, Bio, Shiga, Japan). The obtained cDNA was subjected to PCR using the following procedure: 1 cycle of $5 \mathrm{~min}$ at $94^{\circ} \mathrm{C}$, then 30 cycles of $45 \mathrm{sec}$ at $94^{\circ} \mathrm{C}$, of $45 \mathrm{sec}$ at $59^{\circ} \mathrm{C}$ and of $30 \mathrm{sec}$ at $72^{\circ} \mathrm{C}$. The primer sequences for FOXL1 were designed as described by Qin et al (11). The GAPDH expression was used as an endogenous control.

Cell viability. Cell viability was measured using the 3-(4,5-dimethylthiazol-2-yl)-2,5-diphenyltetrazolium bromide (MTT) assay. Briefly, $5 \times 10^{3}$ of transfected cells were collected and cultured in each well of 96-well plates for 24, 48, 72 or $96 \mathrm{~h}$. The cells were then incubated with $20 \mu \mathrm{l}$ of MTT solutions $(5 \mathrm{mg} / \mathrm{ml})$ for $4 \mathrm{~h}$ at $37^{\circ} \mathrm{C}$. The supernatant was removed and crystals were dissolved in dimethylsulfoxide (DMSO). The absorbance of each well was measured at $570 \mathrm{~nm}$ with the use of an automated microplate reader. The cell viability was calculated using the formula: (OD of treated groups/OD of control group) $\mathrm{x} 100 \%$.

Xenograft tumor experiments. To assess the effects of FOXL1 expression on cell proliferation in vivo, a xenograft model in nude mice was established using U-2 OS cells. Twenty-four sixweek-old specific pathogen-free female athymic (nu/nu) nude mice were purchased from the Shanghai Laboratory Animal Center of the Chinese Academy of Sciences. The animals were kept in cages and provided with clean water and food. The U-2 OS cells that lacked the FOXL1 expression were stably transfected with pcDNA-FOXL1 or pcDNA3.1 and subjected to the established xenograft model. Briefly, $1 \times 10^{6}$ of U-2 OS cells were injected into the right flanks of the nude mice. When xenograft tumors were visible and the volume reached $\sim 0.5 \mathrm{~cm}^{3}$, tumor volumes were measured every three days and calculated using the formula: volume = length $\mathrm{x}$ width ${ }^{2} \mathrm{x} \pi / 6$. The mice were sacrificed after two weeks of observation and the tumor weight was measured. The animal experiments were approved by the Ethics Committee of The First Affiliated Hospital of Bengbu Medical College and efforts were made to minimize suffering.

Cell-cycle analysis. The distribution of the cell cycle was examined and analyzed using flow cytometry. Following transfection with pcDNA-FOXL1, the U-2 OS cells were collected and washed with cold PBS, and fixed with cold $70 \%$ ethanol overnight at $4^{\circ} \mathrm{C}$. The fixed cells were then washed twice with cold PBS and centrifuged at $500 \mathrm{~g}$ for $5 \mathrm{~min}$. The cells were incubated with $10 \mu \mathrm{g} / \mathrm{ml}$ RNase A and $50 \mu \mathrm{g} / \mathrm{ml}$ PI at $37^{\circ} \mathrm{C}$ for $30 \mathrm{~min}$ in the dark. The cells were then analyzed by flow cytometry and the cell cycle was analyzed using FlowJo software. 
A

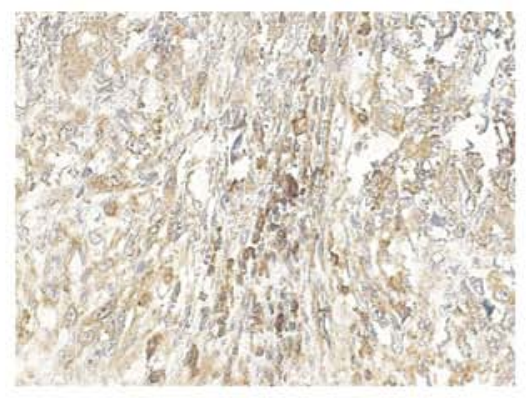

C
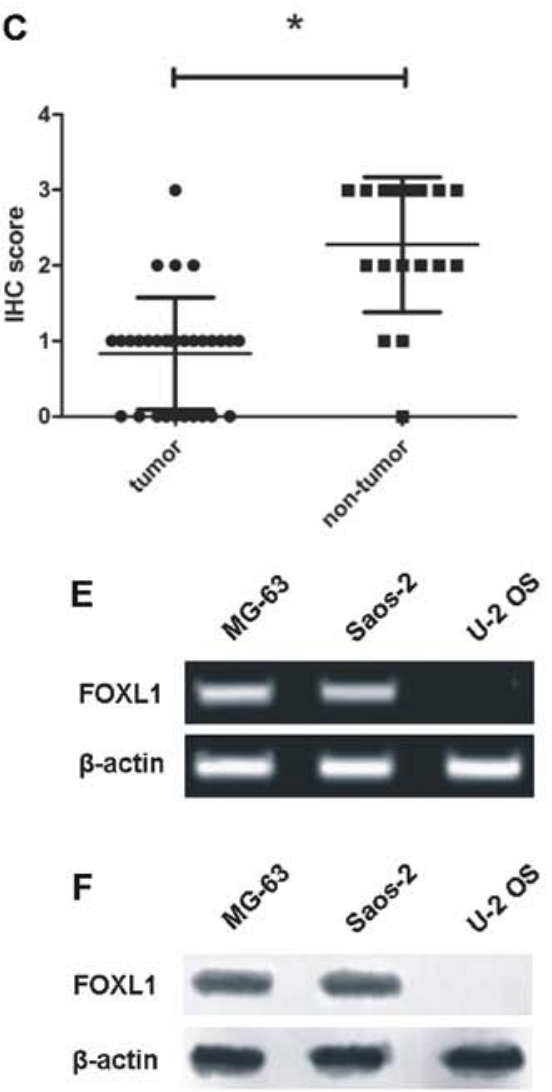

B
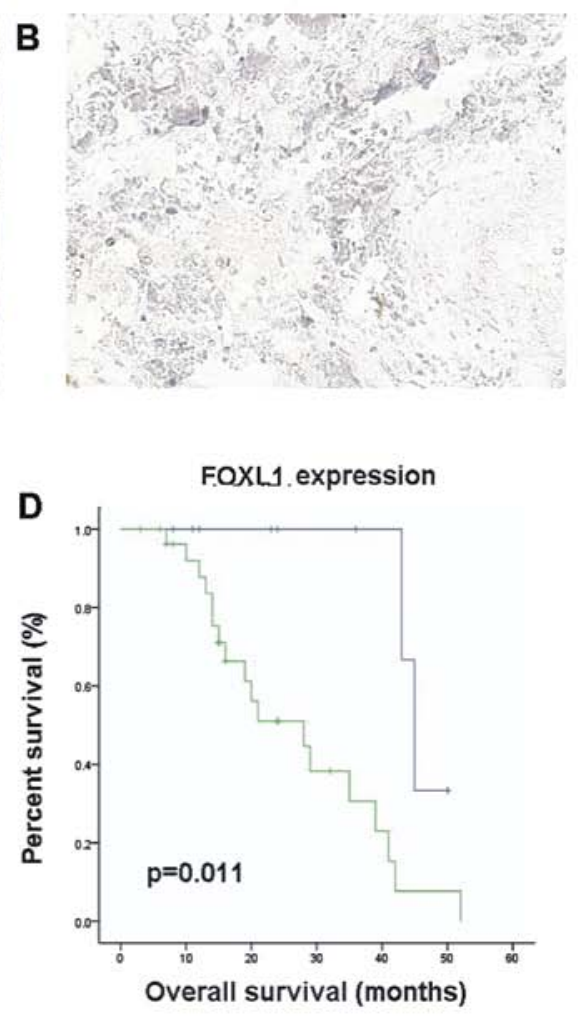

Figure 1. FOXL1 expression in osteosarcoma tissues and cell lines. Immunohistochemical analysis of FOXL1 in (A) normal and (B) osteosarcoma tissues. (C) IHC staining score of FOXL1 in non-tumor tissues was much higher than that in osteosarcoma tissues $(\mathrm{P}<0.05)$. (D) The Kaplan-Meier curves show that the patients with a high expression of FOXL1 had a longer survival rate than those with a low expression of FOXL1 (P<0.05). (E) The mRNA expression of FOXL1 in the MG-63, Saos-2 and U-2 OS cell lines. (F) The protein expression of FOXL1 in the MG-63, Saos-2 and U-2 OS cell lines. (G) Transfection with pcDNA-FOXL1 restored the expression of FOXL1 in the U-2 OS cells. FOXL1, forkhead box L1.

Flow cytometric analysis of apoptosis. Annexin V-FITC staining was performed to detect apoptosis of the U-2 OS cells. Briefly, the transfected cells were collected at a specific time point $(24,48$ and $72 \mathrm{~h}$ ), washed with PBS and then stained with Annexin V-FITC and PI at room temperature for $15 \mathrm{~min}$. The percentage of apoptotic cells was then analyzed by flow cytometry.

Flow cytometric analysis of the mitochondrial membrane potential. The fluorescent probe JC-1 (5,5',6,6'-tetrachloro1,1',3,3'-tetraethyl-benzimidazolo-carbocyanine iodide) was used to measure the depolarization of the mitochondrial membrane potential in the U-2 OS cells. Briefly, the transfected cells were seeded in 6-well plates and were harvested at 24,48 and $72 \mathrm{~h}$. Approximately $1 \times 10^{6}$ cells were incubated with $1 \mu 1$ of $\mathrm{JC}-1(2 \mathrm{mg} / \mathrm{ml})$ at $37^{\circ} \mathrm{C}$ for $20 \mathrm{~min}$. The cells were then washed with PBS twice, followed by FACS analysis (Becton-Dickinson, Franklin Lakes, NJ, USA).

Western blot analysis. The expression of cell cycle- and apoptosis-related proteins was examined using western blot analysis. Briefly, $48 \mathrm{~h}$ after transfection, total proteins were extracted from the U-2 OS cells. The protein $(30 \mu \mathrm{g})$ was subjected to $10-12 \%$ sodium dodecyl sulfate-polyacrylamide gel electrophoresis (SDS-PAGE) and electrotransferred to polyvinylidene difluoride (PVDF) membranes (Millipore, Billerica, MA, USA). The membranes were incubated in blocking buffer and probed with appropriate primary 

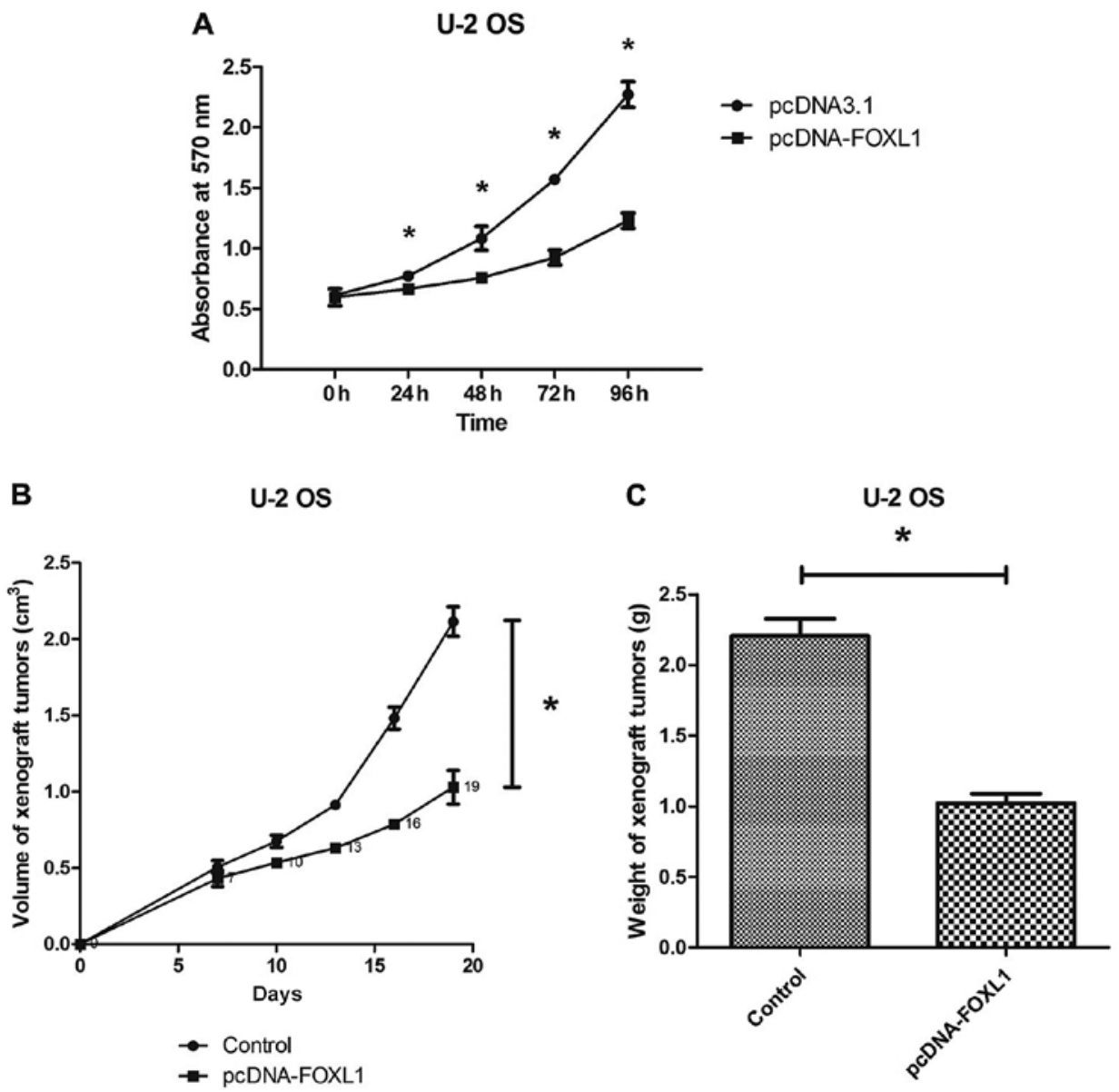

Figure 2. Restoration of FOXL1 expression inhibits U-2 OS cell growth in vitro and in vivo. Restoration of FOXL1 expression significantly inhibits the (A) proliferation of $\mathrm{U}-2$ OS cells $(\mathrm{P}<0.05)$, and $(\mathrm{B}$ and $\mathrm{C})$ the volume and weight of the xenograft tumor $(\mathrm{P}<0.05)$. FOXL1, forkhead box L1.

antibodies (Santa Cruz Biotechnology, Inc.) overnight at $4^{\circ} \mathrm{C}$, followed by incubation with horseradish peroxidase-conjugated secondary antibodies (Santa Cruz Biotechnology, Inc.). The blots were incubated with an enhanced chemiluminescence solution (ECL) kit (Pierce, Rockford, IL, USA) and exposed to X-OMAT Blue film (Kodak, Rochester, NY, USA). Cytochrome $c$ oxidase subunit IV (Cox IV) and $\beta$-actin were used as controls for mitochondrial and cytosolic proteins, respectively.

Statistical analysis. Statistical analysis was performed with SPSS 17.0 (SPSS, Inc., Chicago, IL, USA). The Student's t-test, one-way ANOVA, Kaplan-Meier, log-rank and Chi-square tests were used to analyze the differences between the groups. $\mathrm{P}<0.05$ was considered to indicate a statistically significant difference.

\section{Results}

FOXL1 expression is downregulated in the osteosarcoma tissues and cell lines. Immunohistochemical staining revealed that FOXL1 expression was downregulated in the osteosarcoma tissues. Brown in the nucleus (FOXL1 staining) indicated that the FOXL1 protein was located in the nucleus of normal and cancer cells (Fig. 1A and B). The staining scores of FOXL1 expression in osteosarcoma cases mainly ranged between 0 and 1 , while those in the corresponding normal tissues mainly range from 2 to 3 . The mean score of FOXL1 expression in osteosarcoma tissues was reduced as compared to that in the normal tissues $(\mathrm{P}<0.05$, Fig. $1 \mathrm{C})$. In addition, the patients with a high expression of FOXL1 had a longer survival rate, than those with a low expression of FOXL1 (P<0.05, Fig. 1D).

RT-PCR and western blot analysis revealed that the MG-63 and Saos-2 cell lines had endogenous FOXL1 mRNA and protein, while the U-2 OS cell line lacked endogenous FOXL1 mRNA and protein (Fig. 1E and F). Thus, the U-2 OS cell line was selected for subsequent studies. Additionally, FOXL1 expression in the U-2 OS cell line was restored by transfection with pcDNA-FOXL1 (Fig. 1G).

Restoration of FOXL1 expression inhibits U-2 OS cell growth in vitro and in vivo. The MTT assay showed that transfection with pcDNA-FOXL1 significantly inhibited the U-2 OS cell growth in vitro and this growth inhibition was time-dependent (Fig. 2A). The in vivo inhibitory effect of FOXL1 against the U-2 OS cells was evaluated by a xenograft tumor model. It was observed that the ectopic FOXL1 expression significantly inhibited the growth of xenograft tumors on nude mice. The volume and weight of the FOXL1-overexpressing xenograft tumors were much less than those of the control xenograft tumors (Fig. 2B and C). The in vitro and in vivo studies 

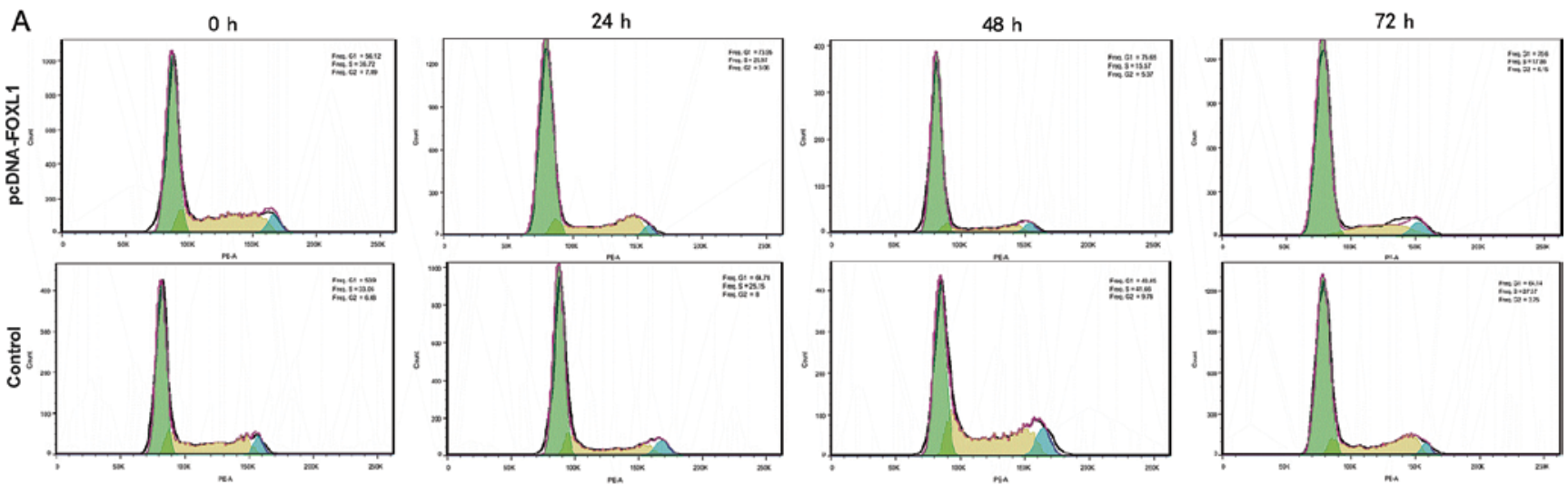

B

U-2 OS

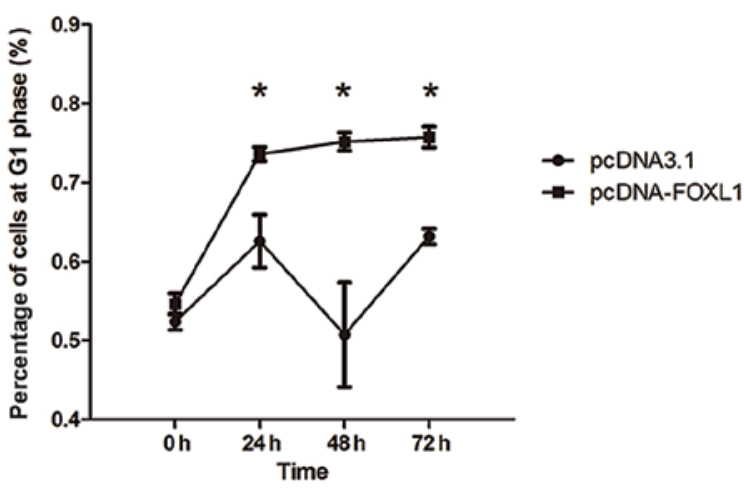

Figure 3. Restoration of FOXL1 expression induces G1 arrest in vitro. (A) Distribution of the cell cycle was analyzed by FlowJo software. The results shown are representative of three experiments. (B) The proportion of U-2 OS cells at G1 phase were significantly increased following transfection with pcDNA-FOXL1 (24-72 h). FOXL1, forkhead box L1.
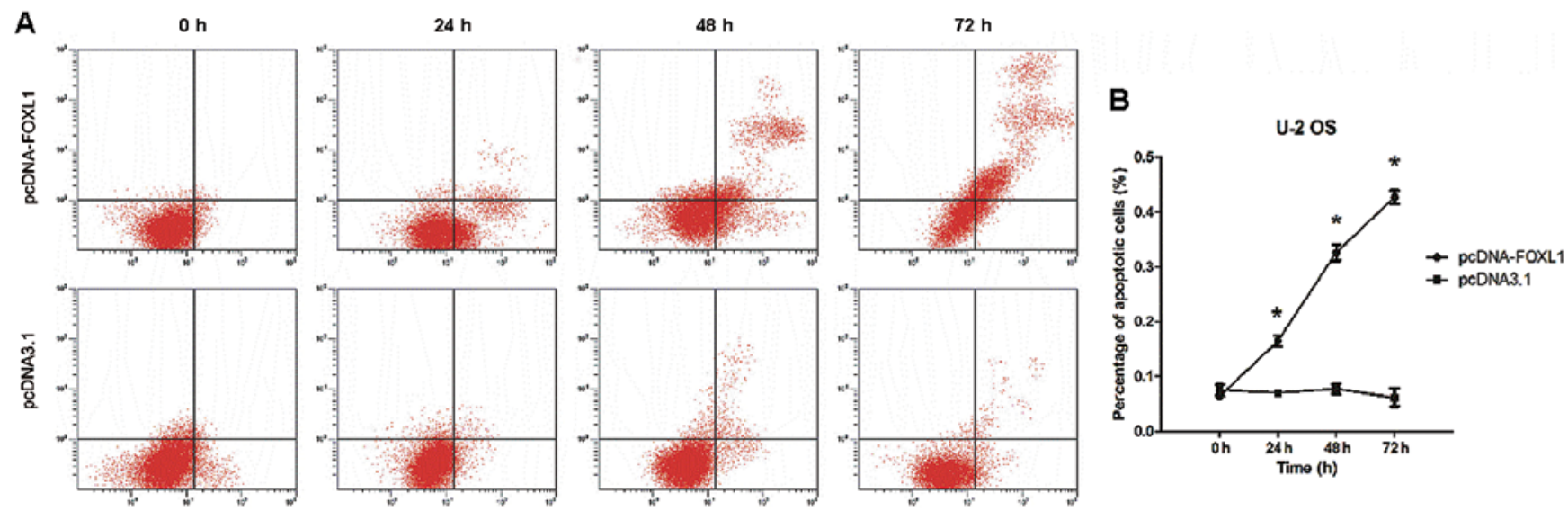

Figure 4. Restoration of FOXL1 expression induces apoptosis in vitro. (A) The viable U-2 OS cells are shown at the lower left quadrant. Cells undergoing early apoptosis are shown at the lower right quadrant. Cells in the end stage of apoptosis or undergoing necrosis are shown at the upper right quadrant. (B) The apoptotic U-2 OS cells were significantly increased following transfection with pcDNA-FOXL1 ( $<<0.05$, between 24 and $72 \mathrm{~h}$ ). Late apoptotic cells were gradually increased between 24 and $72 \mathrm{~h}$. FOXL1, forkhead box L1.

demonstrated that restoration of the FOXL1 expression inhibited the proliferation of the U-2 OS cells, which lacked the endogenous FOXL1 expression.

Restoration of the FOXL1 expression induces G1 arrest in the $U-2$ OS cell line. The distribution of the cell cycle was analyzed by FACS. It was noted that the proportion of the U-2 OS cells in G1 phase was significantly increased following transfection with pcDNA-FOXL1 ( $\mathrm{P}<0.05$ between 24 and 72 h) (Fig. 3).

Restoration of FOXL1 expression induces apoptosis in the U-2 OS cell line. Annexin V/PI staining showed that restoration of FOXL1 expression induced the apoptosis of U-2 OS cells in vitro. As shown in Fig. 4A and B, a significant increase 
A
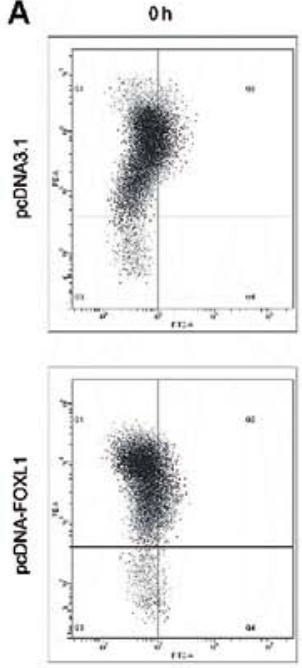

$24 \mathrm{~h}$
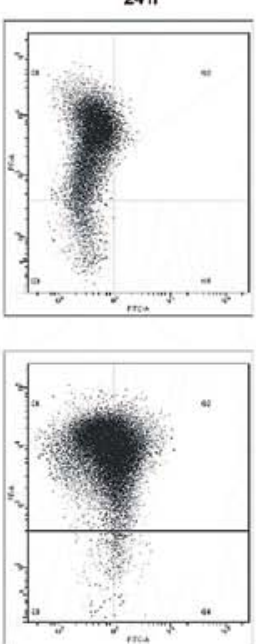

$48 \mathrm{~h}$
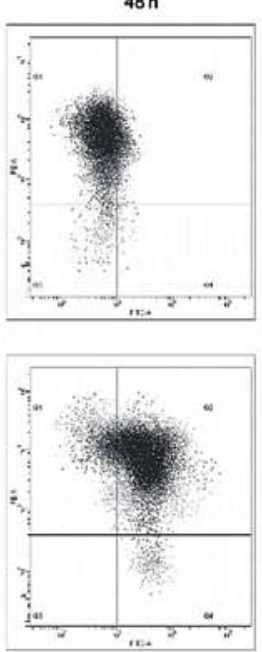
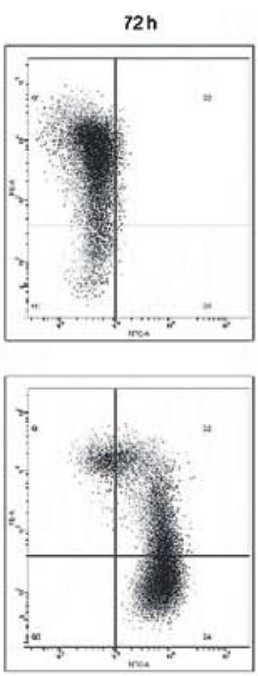

B

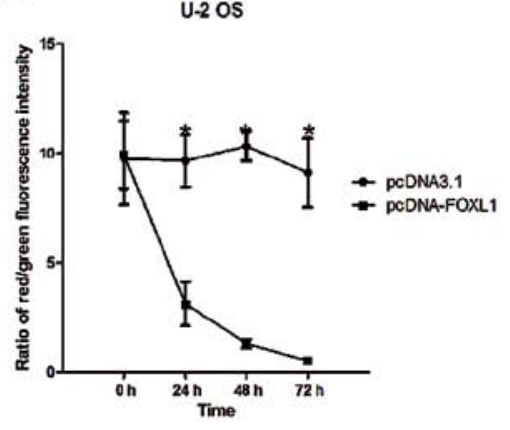

Figure 5. Restoration of FOXL1 expression depolarizes the transmembrane potential depolarization. (A) The loss of the mitochondrial membrane potential $(\Delta \Psi \mathrm{m})$ at $0,24,48$ and $72 \mathrm{~h}$ following transfection was measured by the red/green fluorescence intensity ratio. The $\mathrm{x}$-axis shows the green fluorescence intensity, while the $y$-axis shows the red fluorescence intensity. The decrease of the ratio indicates loss of $\Delta \Psi \mathrm{m}$. (B) Loss of $\Delta \Psi \mathrm{m}$ was observed following transfection with pcDNA-FOXL1 (between 24 and $72 \mathrm{~h}$ ). The proportion of the red fluorescence-positive cells decreased, while that of the green fluorescence-positive cells increased. FOXL1, forkhead box L1.
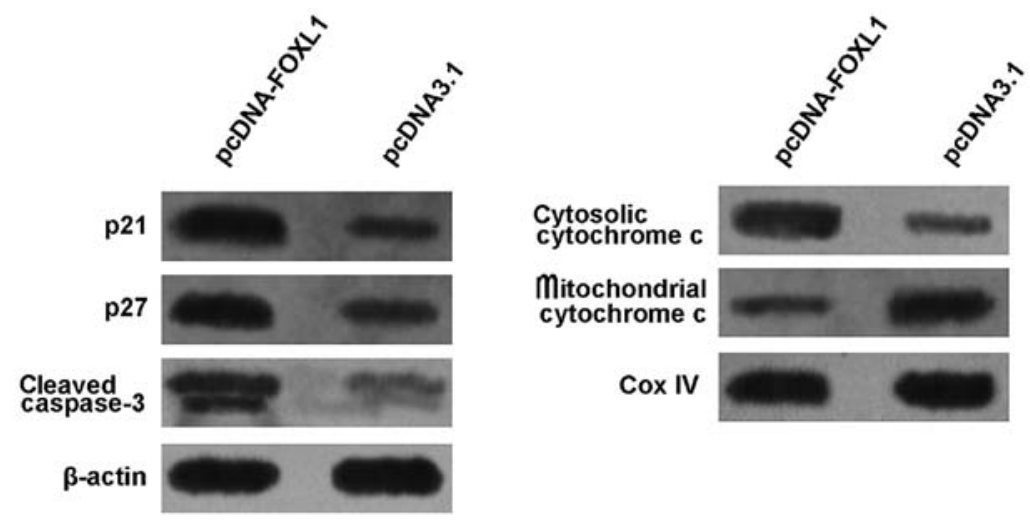

Figure 6. FOXL1 enhances the expression of p21, p27, cytosolic cytochrome $c$ and cleaved caspase-3. FOXL1, forkhead box L1.

in the proportion of apoptotic U-2 OS cells (early plus late apoptosis) was observed following transfection with pcDNAFOXL1 ( $\mathrm{P}<0.0524-72 \mathrm{~h})$. The proportion of late apoptotic or necrotic cells increased in a time-dependent manner (starting from $24 \mathrm{~h}$ and peaking at $72 \mathrm{~h}$ ).

Restoration of FOXL1 expression depolarizes transmembrane potential depolarization. Disruption of the mitochondrial inner transmembrane potential $(\Delta \Psi \mathrm{m})$ is often associated with early apoptosis (20). In the present study, loss of $\Delta \Psi \mathrm{m}$ was determined by the JC-1 staining assay. The shift of JC-1 fluorescence from red to green indicated a collapse of $\Delta \Psi$. As shown in Fig. $5 \mathrm{~A}$ and $\mathrm{B}$, the percentage of low $\Delta \Psi$ cells with JC-1 (green fluorescence-positive) was increased while that of low $\Delta \Psi$ cells with JC-1 (red fluorescence-positive) was decreased, suggesting ectopic FOXL1 expression-induced disruption of the mitochondrial membrane potential of U-2 OS cells.

Restoration of the FOXL1 expression increases the expression of p21, p27, cytosolic cytochrome $c$ and cleaved caspase-3. To preliminarily study the molecular mechanisms for the induction of cycle arrest and apoptosis of the U-2 OS cells following transfection with pcDNA-FOXL1, the expression of p21, p27, cytochrome $c$ and caspase-3 was examined by the western blot analysis. As shown in Fig. 6, the level of cytosolic cytochrome $c$ was markedly increased, while that of mitochondrial cytochrome $c$ was markedly decreased $48 \mathrm{~h}$ after transfection with pcDNA-FOXL1, suggesting that FOXL1 promoted cytochrome $c$ release from mitochondria to cytosol. Furthermore, it was observed that pcDNA-FOXL1 induced an increase in the expression of p21, p27 and cleaved caspase-3.

\section{Discussion}

Gene therapy has been considered as potential strategy for the treatment of osteosarcoma (12). However, the molecular pathogenesis of osteosarcoma-regulating growth and progression remains to be fully elucidated, which may hinder the development of gene therapy. In the present 
study, the role of FOXL1 in the regulation of growth, cell cycle and apoptosis in osteosarcoma cells was preliminarily investigated.

The levels of FOXL1 mRNA and protein were initially examined in osteosarcoma tissues and cell lines. It was demonstrated that the FOXL1 expression was significantly decreased as compared with corresponding non-tumor tissues. Additionally, FOXL1 expression was associated with an improved prognosis in patients undergoing surgery for osteosarcoma. These data indicate the possibility that FOXL1 plays a tumor-suppressor role in osteosarcoma. Similar results were observed in other types of cancer, such as pancreatic and gallbladder cancer $(10,11)$. The regulation of FOXL1 expression remains to be determined, while future studies should be focused on genetic and epigenetic mechanisms for the deregulated FOXL1 expression in tumors.

To identify its role in osteosarcoma, the effects of pcDNAFOXL1 on the proliferation of the U2-OS osteosarcoma cell line lacking the endogenous expression of FOXL1 was subsequently studied. The ectopic expression of FOXL1 inhibited the proliferation of the U-2 OS cells as well as the tumorigenicity and growth of xenograft tumors in vivo. Based on the results of the cell cycle and apoptosis analysis, it was concluded that the antitumor activities of pcDNA-FOXL1 may be attributable to its ability to induce cell cycle arrest and apoptosis.

It has been documented that certain forkhead transcription factors have been shown to play an intrinsic role in controlling cell-cycle progression $(13,14)$. In the present study, it was found that FOXL1 plays a role in controlling G1/S transition. The G1/S checkpoint controls the entry of cells from the first preparation phase (G1) into the DNA synthesis phase (S). A number of cell cycle regulators participate in controlling this checkpoint. $221^{\mathrm{CIP} 1}$ and $\mathrm{p} 27^{\mathrm{Kip} 1}$, which belong to cyclin-dependent kinase inhibitors (CKIs), interact with cyclin-dependent kinases, including CDK2, to inhibit kinase activity and reduce the phosphorylation of the retinoblastoma tumor-suppressor protein $(\mathrm{Rb})(15,16)$. The $\mathrm{Rb}$ complex binds to the E2F-DP1 transcription factors, inhibiting the downstream transcription (17). In the present study, transfection with pcDNA-FOXL1 caused the accumulation of the cyclindependent kinase inhibitors p21 and p27, which may inhibit cdk2 kinase activity and the phosphorylation of $\mathrm{Rb}$, leading to G1 arrest.

During apoptosis, cytochrome $c$ is released into the cytosol as the outer membrane of mitochondria becomes permeable and the mitochondrial transmembrane potential is collapsed, and these events trigger caspase activation and initiation of apoptosis (18). In the present study, it was observed that $\Delta \Psi \mathrm{m}$ was disrupted and cytochrome $c$ was released from mitochondria to cytosol following transfection with pcDNA-FOXL1. In addition, caspase-3, the key executioner in the process of apoptosis (19) was activated. These findings suggest that the ectopic FOXL1 expression activated the intrinsic (mitochondrial) apoptotic pathway.

In conclusion, our results suggest that FOXL1 plays a tumor-suppressor role in osteosarcoma and the restoration of FOXL1 expression by gene therapy providing a potential treatment for osteosarcoma patients lacking endogenous FOXL1 expression.

\section{Acknowledgements}

The present study was supported by the Natural Science Foundation of Bengbu Medical College (No. Byky1340). We thank Dr Qin for his support for the present study.

\section{References}

1. Kim HJ, Chalmers PN and Morris CD: Pediatric osteogenic sarcoma. Curr Opin Pediatr 22: 61-66, 2010.

2. Marina N, Gebhardt M, Teot L and Gorlick R: Biology and therapeutic advances for pediatric osteosarcoma. Oncologist 9: 422-441, 2004

3. Coffer PJ and Burgering BM: Forkhead-box transcription factors and their role in the immune system. Nat Rev Immunol 4: 889-899, 2004

4. Hoeller S, Schneider A, Haralambieva E, Dirnhofer S and Tzankov A: FOXP1 protein overexpression is associated with inferior outcome in nodal diffuse large B-cell lymphomas with non-germinal centre phenotype, independent of gains and structural aberrations at 3p14.1. Histopathology 57: 73-80, 2010.

5. Koon HB, Ippolito GC, Banham AH and Tucker PW: FOXP1: A potential therapeutic target in cancer. Expert Opin Ther Targets 11: 955-965, 2007.

6. Bergamaschi A, Madak-Erdogan Z, Kim YJ, Choi YL, Lu H and Katzenellenbogen BS: The forkhead transcription factor FOXM1 promotes endocrine resistance and invasiveness in estrogen receptor-positive breast cancer by expansion of stem-like cancer cells. Breast Cancer Res 16: 436, 2014.

7. Santo EE, Ebus ME, Koster J, Schulte JH, Lakeman A, van Sluis P, Vermeulen J, Gisselsson D, Øra I, Lindner S, et al: Oncogenic activation of FOXR1 by 11q23 intrachromosomal deletion-fusions in neuroblastoma. Oncogene 31: 1571-1581, 2012.

8. Gong C, Fujino K, Monteiro LJ, Gomes AR, Drost R, Davidson-Smith H, Takeda S, Khoo US, Jonkers J, Sproul D, et al: FOXA1 repression is associated with loss of BRCA1 and increased promoter methylation and chromatin silencing in breast cancer. Oncogene: Dec 22, 2014 (Epub ahead of print). doi: 10.1038/onc.2014.421.

9. Perreault N, Sackett SD, Katz JP, Furth EE and Kaestner KH: Foxll is a mesenchymal Modifier of Min in carcinogenesis of stomach and colon. Genes Dev 19: 311-315, 2005.

10. Zhang G, He P, Gaedcke J, Ghadimi BM, Ried T, Yfantis HG, Lee DH, Hanna N, Alexander HR and Hussain SP: FOXL1, a novel candidate tumor suppressor, inhibits tumor aggressiveness and predicts outcome in human pancreatic cancer. Cancer Res 73: 5416-5425, 2013.

11. Qin Y, Gong W, Zhang M, Wang J, Tang Z and Quan Z: Forkhead box L1 is frequently downregulated in gallbladder cancer and inhibits cell growth through apoptosis induction by mitochondrial dysfunction. PLoS One 9: e102084, 2014.

12. Tan ML, Choong PF and Dass CR: Osteosarcoma: Conventional treatment vs. gene therapy. Cancer Biol Ther 8: 106-117, 2009.

13. Furukawa-Hibi Y, Yoshida-Araki K, Ohta T, Ikeda K and Motoyama N: FOXO forkhead transcription factors induce $\mathrm{G}_{2}-\mathrm{M}$ checkpoint in response to oxidative stress. J Biol Chem 277: 26729-26732, 2002.

14. Kazantseva YA, YarushkinAAand Pustylnyak VO: CAR-mediated repression of Foxo1 transcriptional activity regulates the cell cycle inhibitor p21 in mouse livers. Toxicology 321: 73-79, 2014

15. LaBaer J, Garrett MD, Stevenson LF, Slingerland JM, Sandhu C, Chou HS, Fattaey A and Harlow E: New functional activities for the p21 family of CDK inhibitors. Genes Dev 11: 847-862, 1997.

16. Møller MB: P27 in cell cycle control and cancer. Leuk Lymphoma 39: 19-27, 2000.

17. Giacinti $\mathrm{C}$ and Giordano A: RB and cell cycle progression. Oncogene 25: 5220-5227, 2006.

18. Jiang X and Wang X: Cytochrome $c$-mediated apoptosis. Annu Rev Biochem 73: 87-106, 2004.

19. Porter AG and Jänicke RU: Emerging roles of caspase-3 in apoptosis. Cell Death Differ 6: 99-104, 1999.

20. Ly JD, Grubb DR and Lawen A: The mitochondrial membrane potential (deltapsi(m)) in apoptosis; an update. Apoptosis 8: 115-128, 2003. 